\title{
Error-driven learning of communication systems in biological life forms
}

\author{
Jessie S. Nixon, Fabian Tomaschek \\ jessie.nixon@uni-tuebingen.de, fabian.tomaschek@uni-tuebingen.de \\ Quantitative Linguistics Group, Department of General Linguistics, \\ University of Tübingen, Germany
}

Learning processes in Earth's animal species have been studied in depth for over a century, from which have emerged mathematical, theoretical and computational error-driven models of learning. Despite a great deal of variation in biological systems, error-driven learning models have been successful in predicting learning behaviour, including humans and language. We propose that errordriven learning is likely to also play a role in the perception and communication systems of extraterrestrial intelligence. We examine how some very minor changes in conditions could effect the characteristics of an acoustic communication system. We then simulate the effects of learning on Earth vs. extra-terrestrial conditions and how this would affect perception of the respective communication signal.

\section{Introduction}

The goal of this volume is to contemplate what possible languages or communication systems might be used by interstellar life forms, with the aim of creating some insight into how to tackle the task of interpreting communication signals should we ever encounter them. An important aspect of understanding how a communication system works is to understand how it is formed; that is, how it is learned, how it is passed on between generations. The answers to this question also have implications for potential cross-species communication. There are obvious challenges to this task, given that when it comes to communication, we have no data other than from our own species and other species on Earth, which are all genetically related. Nevertheless, we can speculate on the possibility that other interstellar intelligence might use similar learning systems to those used on Earth, but with substantially different biological systems and operating in different physical environments, about the latter of which we have some basic data.

We start by outlining the scale of the problem facing learners of human languages. Humans use the speech signal to discriminate concepts, such as 'house' or 'mouse' and 'love' or 'fear'. The speech signal is mindbogglingly complex and contains a huge amount of non-discriminative 
information or noise - that is, signal that does not help to understand the message (for sources of noise in the speech signal, see Altmann, 1980; Fosler-Lussier and Morgan, 1999; Fuchs et al., 2008; Gay, 1978; Hirata, 2004; Leemann et al., 2012; Tomaschek and Leemann, 2018; Tomaschek et al., 2013, 2014, 2018a, b; Weirich and Fuchs, 2006; Winkler et al., 2006) . Therefore, learning a human language requires learning mechanisms that can adequately handle this level of both complexity and noise in the system. We then present an overview of error-driven learning, the learning mechanism used across the board by Earth's animal (and possibly also plant) species. We examine two specific ways in which the signal of communication systems could vary either due to different biological systems or different atmospheric conditions. Finally, we simulate cross-species signal perception in one specific case where an extra-terrestrial intelligence uses the same articulatory system as humans, but produces a different signal due to body size.

The chances of face-to-face communication with interstellar intelligence in the foreseeable future seem extremely slim. If we ever encounter communication signals from other interstellar species, it is most likely that one of two possibilities occur. Firstly, we might receive some sort of simple hail or other signal designed for the purpose of communicating over long distances, such as electromagnetic pulses (flashes of light). Alternatively, we might encounter artefacts intended as intraspecies communication or similar multimedia signals somehow conveyed to us with the intention of communicating. Examples from Earth would be (overheard) radio waves, television signal or internet transmissions. These two scenarios differ greatly with respect to the type of signal involved and the appropriate response. With respect to the first possibility, if a direct signal were received, we would have no shared code, no code book to look up and select the correct intended message from. So we would either have to ignore it or somehow try to learn the intentions of the signaller.

While this may seem an obvious point, it actually points to a key principle of communication. Communication has two components - the signal or code and the message, the intention of the signaler or world event that it relates to. In learning theory, in the case of learning the code, these components are the cues and the outcomes, respectively (Ramscar, 2019; Ramscar et al., 2017; Rescorla, 1988). This scenario is in some ways similar to the scenario an infant faces. There is a signal in the form of sensory cues, but no established code to determine what they might mean. However, the critical difference is that an infant has a huge amount of constantly incoming information about how the signal predicts events in the world. In the case of a distant and simple interstellar signal, like flashes of Morse code, we are likely to have little opportunity to learn the intended message to any high level of complexity. For example, we might attempt to reply by imitating the light signal and the two sides might make a friendly exchange of returning the same or similar messages back and forth. This would establish contact and would confirm the existence of 
both parties and to some extent the intention of communication. But beyond this point, how would it be possible to establish any further shared code without both parties also sharing specific experiences of the world at that same time? How would certain signal come to be associated with certain events and not others? (See also Slobodchikoff, this volume, for further discussion of this point).

In this chapter, we focus mainly on the learning mechanism by which interstellar intelligence might learn their own communication system and the implications this might have for the life form's perception. In terms of human encounters with such a communication system, this would presumably require at least obtaining access to some sort of multimodal signal in which not only the language itself but also the surrounding events are available. We agree with Slobodchikoff (this volume) that if we had access to the signal alone, we would have little hope of learning much about the communication system other than characteristics of the signal itself. One characteristic of words is their linguistic context ("You shall know a word by the company it keeps", Firth, 1957). Covariance structures between words are modelled with semantic networks which learn that cat and dog are more similar than cat and penguin due to their use in language (Landauer \& Dumais, 1997). However, this only examines structure within the system. We would not have a means to learn the intentions of the signaler, the relation of the signal to the world. However, if the communication contained both signal and sufficient information about how it relates to the world - for example, in some sort of multimodal or even interactive format - we would have a better chance of learning something about the world of the life form and the system they use to communicate about it. ${ }^{1}$ Knox et al. (2019) have achieved this with wild Orang-Utans.

A not insignificant problem will be determining what the culture-specific important world events of the interstellar intelligence are. In human societies, it is common to present images of common objects (plants, animals, houses) and activities (walking, eating) for learning foreign languages. The images are generally recognised across cultures by speakers of different languages. However, this requires shared experience and can quickly break down when culture or technology diverge. Buszard-Welcher (this volume) describes a project that takes on some of these challenges, namely creating an archive of human cultures designed for our own decendents millenia in the future. For extra-terrestrial intelligence, without knowing even what kind of sensory systems they have, it becomes even more difficult to determine what experiences we might have in common, but the question is worth considering. Might they be familiar with concepts such as planets, stars, weather patterns, day and night, water and ice? However, we leave that issue aside for now.

1 We leave aside the technical issue of how the technology is made usable to unfamiliar users. 



\section{Human communication systems}
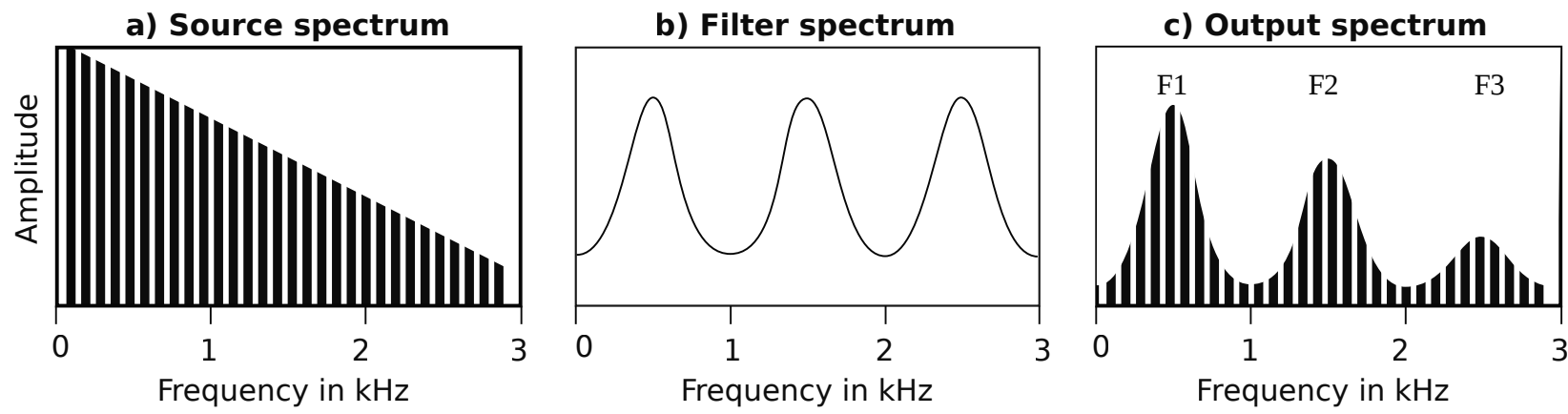

Fig. 1: a) Schematic presentation of the source spectrum created by the vocal cords. b) Filter spectrum of the vocal tract, which functions as a resonance filter. c) Spectrum of the articulated vowel. Each spectral peak represents a formant (F1, F2, F3). Changing the shape of the mouth (i.e. its diameter) changes the relative location of the formants. Plots according to Fant (1960, p. 19).

Human communication systems come in diverse forms and are extremely complex. Some examples of communication systems that have emerged through pressure for communication are sign language (Nyst, 2013, 2015), gestures, visual cues such as facial expression and body language, drum languages (Rattray, 1923) and whistling languages (Meyer, 2004). ${ }^{2}$ This diversity demonstrates a high degree of adaptivity - as long as the sensory input is able to be discriminated to a sufficient level of complexity, it can be used for communication. However, by far the most common form of communication is spoken language.

Human speech is primarily based on an acoustic signal which is created by movements of the human masticatory organs - jaw, lips and the tongue (Weber et al., 1986; Yashiro and Takada, 2005). The acoustic system used by humans is often divided into two broad categories - consonants and vowels. "Consonants" constitute partial or complete constriction of airflow. Constriction of airflow is achieved in various ways, including closing of the jaw, placing various sections of the tongue against different areas of the mouth, closing the lips and sealing the oral cavity to allow airflow through the nasal cavity. Sounds created by an unconstricted airflow are classified as "vowels". To produce vowels, the vocal tract functions as a resonance filter that filters the full spectrum signal created by the vocal cords (Figure 1, a \& b). The acoustic signal contains several spectral peaks (Figure 1, c), where the first peak is called the first formant (F1), the second peak the second formant (F2), and so forth. The spectral properties in Figure 1 (c) represent the vowel

2 There also exist consciously designed technologies for communcation, such as braille and other writing systems and code languages - see Harbour, this volume, for a discussion of writing systems. 
schwa, which is articulated by positioning the tongue in the center of the vocal tract and is equivalent to the first vowel in words like "about" or the second vowel in "speaker".

Moving the tongue in the oral cavity modifies the vocal tract's shape, changing the characteristics of the resonance filter, which results in different formant frequencies, thus in different vowel qualities. For example, a high fronted tongue position creates an [i], a lower central tongue position an [a] and a high-retracted tongue position with lip rounding a $[\mathrm{u}](\mathrm{cf}$. Figure 3, a). Figure 3 (b) shows the vowel space on the basis of F1 (y-axis) and F2 (x-axis) frequencies. The black triangle represents the location of the five human standard vowels in the F1/F2 vowel space. Note that the orientation of the triangle in Figure $3(\mathrm{~b})$ is equivalent to the triangle's orientation in Figure 3 (a).

During speech, the various different speech apparatuses move dynamically, leading to continual changes to the resonance filter. This means that, for example, the vowel in "bat" is different to the vowel in "can", because both preceding and following mouth shapes affect the resonance filter (Magen, 1997; Öhman, 1966). If clustered by sound category, cue values vary around a centre with considerable overlap between clusters. In other words, rather than being discrete, with a consistent mapping to semantics or even to sound categories, acoustic cues are gradient and variable.

\section{How is it possible for such a complex system to be learned?}

Although speech can be an effective means of communication, the speech code is so complex that after decades of study, on an academic level we still understand neither what cues make up the system nor the relationship between auditory cues and semantics or the messages being conveyed. Some researchers propose that speech is made up of a small set of discrete sound units (sometimes called "phonemes"). However, if people are asked to identify words taken from recordings of running speech, accuracy is as low as $20 \%-40 \%$ by participant (Arnold et al., 2017). If speech consisted of strings of sound units, we would not expect such difficulties when these individual units are extracted from speech. Contrast this with written language: a single word can generally still be read with no additional difficulty when extracted from a sentence.

A large body of evidence has accumulated over the last several decades showing that human infants and adults are highly sensitive to the distributional characteristics of cues in speech (Clayards et al., 2008; Maye et al., 2002; McMurray et al., 2009; Nixon and Best, 2018; Nixon et al., 2016, 2018; Yoshida et al., 2010). This line of research has been groundbreaking, because it has demonstrated that humans have sufficient learning capabilities to learn speech and language from 
the input. Prior to this, many researchers believed that language was too complex to learn and must therefore be innate. However, while these data are often interpreted as suggesting that learning occurs by directly learning the statistical structure through statistical clustering mechanisms (e.g. Maye et al., 2002), computational models have demonstrated that unsupervised, purely statistical mechanisms cannot account for learning of speech sounds (McMurray et al., 2009) and recent findings demonstrate that the data are better accounted for with error-driven discriminative learning (Nixon, accepted; Olejarczuk et al., 2018; Ramscar et al., 2013).

According to information theory, communication boils down to the reproduction of a message at one point from a message sent at another point (Shannon, 1948). There is a set of shared codes that point to a limited set of predefined messages. "Comprehension" of a message simply means selecting the correct message from all the possible messages that the sender could have sent. If we consider international Morse code prosigns, this makes sense intuitively. Probably the most famous example is - - - - - - -, a series of three short, three long then three short signals. This code signals a distress call in the case where there is an imminent threat to life or property. It is distinct from, for example, a general call for attention or hail - - - - - or from a request for repetition - - - - - or "correction, my preceding message was an error" - - - - - - - The messages do not break down into components that "contain meaning"3 (see Ramscar, et al., 2010; Wittgenstein, 1953, for discussion of the problems around meaning and reference). Instead, communication of this message from the sender to the receiver is possible because both parties share the same code and this code allows the receiver to discriminate between a distress call, a request for repetition, an error correction and any other possible message. Based on the code, the receiver can select which of the possible messages was intended.

As discussed above, if a direct signal were received from interstellar life, perhaps in the form of light signals akin to flashes of Morse code, we would have no shared code, no code book to look up and select the correct intended message from. So we would either have to ignore it or somehow try to learn the intentions of the signaller.

There is good reason to believe that natural languages behave in the same way as the Morse code example above. "Understanding" a message is a matter of listeners using signals (cues) sent by the speaker to select or discriminate between a finite set of possible intended messages (Ramscar et al., 2010). In humans, the shared code is developed over a lifetime through interaction with the world and with other users of the communication system. The code is constantly negiotiated between sender and receiver. One consequence is that no two users have identical code, so it is only

3 Though the signal can be transcribed with several alternative letter combinations, the most common is 'SOS', which has unofficially come to be associated with the phrase 'save our souls'. 
partially shared (Ramscar, 2019). One theory to explain how a shared code develops is error-driven learning.

Error-driven learning is a learning system used by perhaps all animal species on Earth (Waelti et al., 2001; Mantague et al., 2004; Soto \& Wassermann, 2010) . Learning processes in organisms on Earth, especially animal species, have been meticulously studied for over a century, from which well-developed, well-tested mathematical, theoretical and computational models of learning have emerged (Rescorla and Wagner, 1972; Widrow \& Hoff, 1960). Despite enormous surface variation in biological systems, error-driven learning models have successfully predicted learning behaviour across a broad range of species and under a wide variety of conditions, including humans and language. What these studies demonstrate is that in biological systems on Earth, learning involves using incoming sensory information, or cues, to predict important events in the world. When cues frequently predict a certain outcome, then experiencing that cue again in the future leads to an expectation that the outcome will also occur. Error-driven learning models have been remarkably successful in predicting and explaining a broad range of phenomena in human language, including language acquisition, comprehension and production (Arnold et al., 2017; Baayen et al., 2017, 2019; Nixon, 2018, accepted). The pervasiveness of error-driven learning as a pattern of learning behaviour across such disparate species on Earth makes it a strong candidate as a means of learning among other interstellar species as well. This is not a given, of course. Since all species on earth are genetically related, it is possible that we may have a genetic predisposition to error-driven learning that does not occur in interstellar intelligence. It may also be worth pointing out that error-driven learning is not the sole learning mechanism used among Earth's species. Nevertheless, it is a good starting point and a reasonable hypothesis that other species use similar learning mechanisms.

\section{Error-driven learning in non-human animals}

Perhaps the most famous learning experiments are Pavlov's experiments with dogs at the turn of the $20^{\text {th }}$ century. Pavlov found that if he repeatedly presented a stimulus, such as tone or a light, before feeding the dogs, eventually the dogs would start salivating in response to the stimulus even when the food was not present. Initially this was considered a simple reflex response and thought to be merely due to contiguity of the stimuli: the reflex response that belonged to the food somehow got taken over by the new stimulus (e.g., Hull, 1943; Spence, 1956; reviewed in Gluck \& Bower, 1988). However, in the decades that followed, it became clear that, rather than a simple association, the animals were using information in the cues to learn about the world through a process of prediction and feedback. 

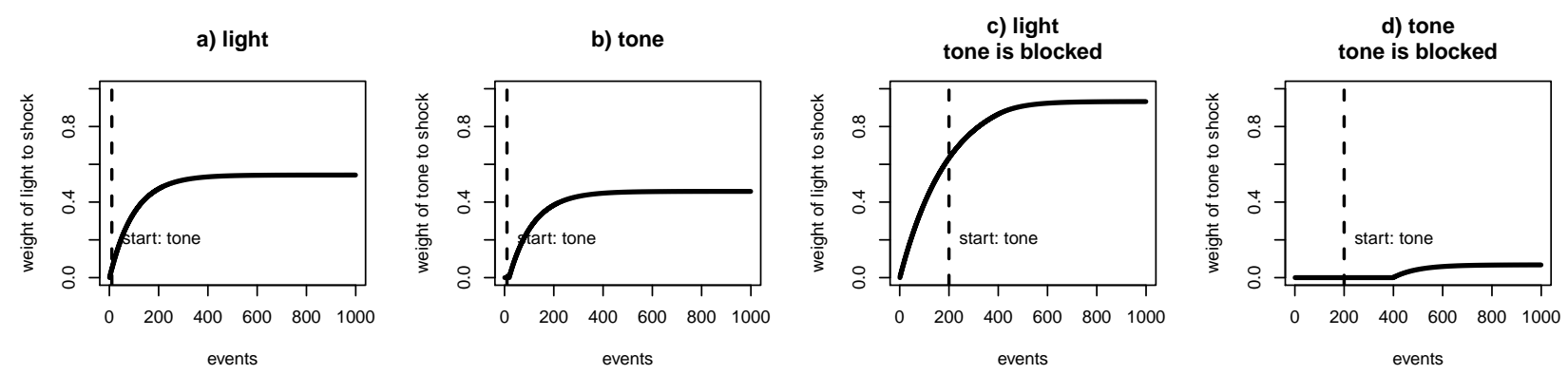

Fig. 2: Illustration of the blocking effect estimated by means of Rescorla-Wagner learning equations. $a \& b)$ Light and tone a cues for shock are trained together. $c \& d)$ Light is pretrained, tone is blocked. Vertical dashed line indicates the beginning of training with the tone cue in addition to the light cue.

In a seminal study, Kamin (1968) trained rats with two different cues, a light and a tone, followed by an electric shock (see Figure 2). In the test, if he presented just one of these cues, say the tone, the rats would exhibit a fear response to the tone - they had learned that the tone predicted the shock. A separate group of rats had identical training and test, except that before the light + tone training, there was a pre-training phase in which the rats were trained with only one cue - the cue that was not tested - in this case, the light. The pre-trained rats did not respond to the tone. Because the light already provided sufficient information to predict the shock, the tone added no additional predictive value. There was no longer sufficient uncertainty left to drive learning of the tone. So the tone wasn't learned. In other words, learning of the tone was blocked by the already-learned light cue.

The Rescorla-Wagner equations were developed to account for this finding as well as a wealth of data that had accumulated over the previous decades from research on animal learning. The level of expectation of a particular outcome given a particular cue or cues is described as the cue-outcome connection weight. Connection weights are adjusted in each learning event. The mathematical equations can be boiled down to a few simple principles. Firstly, for any cues not present in a given learning event, there is no change to the connection weights. For cues present during a learning event connection weights are 1) increased to all outcomes that occur and 2) decreased to any outcomes that do not occur, but which have been encountered previously. What is important is that the amount of change in 1) and 2) depends on the history of learning. When an outcome is highly expected (that is, connection weights are strong due to previous learning), and the outcome occurs, there is little surprise and therefore little learning, so although connection weight increases, the increase is relatively small. In contrast, if an outcome is not expected (that is, 
connection weights are weak, because for example, the cues have not been encountered before or the cues have been encountered before, but the outcome did not occur), but the outcome does occur, the surprise is greater and therefore learning is greater. The increase in connection weights is greater than in the former example.

These simple equations make strong predictions that have been powerful tools for understanding learning behaviour. For example, learning is non-linear and current learning depends on the history of learning. This leads to the famous "learning curve" - the idea that learning is fast in the beginning then tapers off as things become predictable.

\section{How the speech of interstellar intelligence might sound}

Although human speakers are unaware of this, the acoustic characteristics of their speech signal depend on the air temperature and density, which affect the speed of sound, $c$, and their body size, which affects the size of their vocal tract $L$. These variables can be used to calculate the formant frequencies of [@] and therefore all other vowels using equation (1)

(1) $F n=(2 n-1) c / 4 L$,

where $\mathrm{n}$ represents the formant's number, $c$ the speed of sound, and $L$ the length of the vocal tract. For an average human vocal tract whose length is $L=17.5 \mathrm{~cm}$ and the average speed of sound $c=$ $350 \mathrm{~m} / \mathrm{s}$ (with air density $\sim 1.20 \mathrm{~kg} / \mathrm{m}$ and $20^{\circ} \mathrm{C}$ ), F1 $=500 \mathrm{~Hz}$ and F2 $=1500 \mathrm{~Hz}$. The human vowel space in Figure 3 is based on these measures. Figure 3When temperature on Earth drops to $2{ }^{\circ} \mathrm{C}$ at sea level, speed of sound is slower $(\mathrm{c}=319 \mathrm{~m} / \mathrm{s})$, directly affecting formant frequencies produced by the same human speaker. Looking at the differences between the planets in our own solar system in terms of their atmospheric composition and therefore speed of sound (NASA), it is very unlikely that the atmosphere of the interstellar life form's home planet will have exactly the same physical characteristics as our own. For example, speed of sound on Mars ranges from 244 $\mathrm{m} / \mathrm{s}$ at ground level to $220 \mathrm{~m} / \mathrm{s}$ at the top of Olympus Mons at an altitude of $24 \mathrm{~km}$ (NASA). Since speed of sound will be proportional to the density and temperature of the life form's planet, the spectral characteristics of the acoustic signal of interstellar life forms will vary, too, if they communicate by means of acoustic signals.

Let us inspect what effect variation in speed of sound has on vowel acoustics, if, as improbable as this might seem, interstellar life forms create an acoustic signal in the same way as humans do and if their physiology is similar to ours (as is very often suggested by science fiction movies and books). To illustrate effects of the atmospheric properties of the life form's home planet, we can manipulate the $\mathrm{c}$ parameter in equation (1). The greater $\mathrm{c}$ at the extraterrestrials life form's planet, the higher the formant frequencies, significantly affecting the vowel quality of the produced sound. 

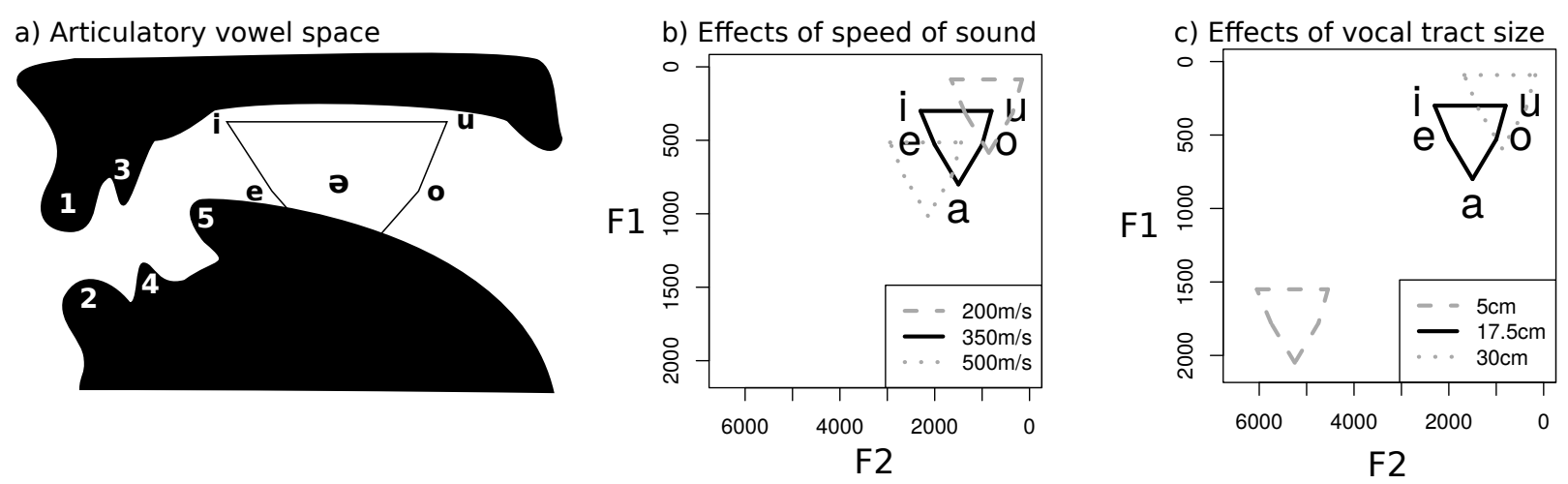

Fig. 3: a) Midsagittal cut through the mouth. $1 \& 2=$ lips, $3 \& 4=$ teeth, $5=$ tongue tip. The black triangle represents the location of the tongue body when articulating the respective vowels. b) Effects of vocal-tract length onto the spectral properties of the vowel space. Black triangle indicates the human vowel space; the grey triangles indicate how vowels produced by longer and shorter vocal tracts are perceived by human ears. Note that frequencies are inverted on both axes to mirror the orientation of the vowel space with that in the mouth. c) Effects of the speed of sound. Black triangle represents the human vowel space, and grey triangles represent what the same articulatory targets would sound like in atmospheres with faster or slower speeds of sound.

Life forms that live on a planet whose atmosphere has $\mathrm{c}=200 \mathrm{~m} / \mathrm{s}$ will create vowels that have frequencies lower than human vowels (grey dashed triangle in Figure 3, b). An [a] produced by the interstellar intelligence will thus sound to human ears like an [o]. When the speed of sound is higher than on Earth, e.g. $\mathrm{c}=500 \mathrm{~m} / \mathrm{s}$, the life form will produce vowels whose formants are on average higher (grey dotted triangle in Figure 3, b). This means that the interstellar's [u] will sound like a [@], an [o] will sound like an [a]. As a side note, similar effects can be achieved when humans speak in a helium-saturated atmosphere (MacLean, 1966).

Variation of air density and temperature all around Earth result in minimal, but measurable variations of the quality of these vowels, making a one-to-one mapping between sound and category as well as meaning impossible. Furthermore, atmospheric characteristics on Earth can vary on a daily basis, which is why humans need to learn to account for these changes. On earth, this is the case with crickets. The frequency of the cricket's signal varies with the ambient temperature and female listeners adapt to the temperature dependent changes (Doherty, 1985). Thus, the interstellar intelligence, even if they create a signal by other means than a wind pipe (Fant, 1960), will have to have a learning mechanism to adapt to changes based on the physical properties of their home planet's atmosphere.

Even if these life forms do create a communication signal by means some kind of wind pipe similar to the human vocal tract, it is still very likely that they will differ in their size from humans, and so will their vocal tract. In other words, the communication signal will depend on the life 
form's physiology. Evidence for this idea comes from the calls of prairie dogs — rodents roughly $30 \mathrm{~cm}$ in height when standing - which indicate the presence of various predators by means of alarm calls. Like humans, they create calls by means of their masticatory apparatus, manipulating the acoustic quality of their calls (Slobodchikoff and Placer, 2006; Slobodchikoff, this volume). While the spectral characteristics of prairie dog calls strongly resemble those of vowels, the formants of these sounds consist of higher frequencies.

We can illustrate the effect of body size, and therefore vocal tract size, by manipulating the parameter $\mathrm{c}$ in equation (1). In contrast to speed of sound, the size of the vocal tract has very strong effects on the acoustic characteristics of vowels if we assume $\mathrm{c}=350 \mathrm{~m} / \mathrm{s}$ (Figure 3, c). Interstellar life forms whose vocal tract is significantly shorter than the average human vocal tract, e.g. $5 \mathrm{~cm}$ long, will produce formant frequencies significantly higher than human formant frequencies. F1 would fall into the range $1500 \mathrm{~Hz}$ to $2000 \mathrm{~Hz}, \mathrm{~F} 2$ would fall into the range of $4500 \mathrm{~Hz}$ to $6000 \mathrm{~Hz}$ (grey dashed triangle in Figure 3,c). These frequencies are still within the range of human perception. Although F1 values are within the range that humans use for speech, the F2 values are higher than the frequencies used in any vowels in human speech., Therefore all sounds will sound strange to human ears, independently of which vowel type (i.e. vocal tract shape) these life forms produce. Because of the high F1 and F2 values, the signal will be most similar to and subjectively sound most like a mix between human [a] and [e] vowels (for which F1 are located between $500 \mathrm{~Hz}$ and $1000 \mathrm{~Hz}$ and F2 are located between $800 \mathrm{~Hz}$ and $2500 \mathrm{~Hz}$ ). Life forms who have longer vocal tracts than the average human will produce vowels with lower formant frequencies (grey dotted triangle in Figure 3, c). Supposing a vocal tract of length $30 \mathrm{~cm}$, the lower part of the vowel space of these beings will overlap with the upper part of the human vowel space. To produce an [a], humans lower the jaw and move the tongue a little bit forward. If life forms with a longer vocal tract performed the same articulatory gesture, the physical signal would be equivalent to (and would sound to humans like) a human [o]; similarly, an [o] would sound like an [u].

Above, we discussed what vowel like sounds will sound like if body size and therefore the vocal tract size were different from humans. These assumptions were based on adult speakers. However, the size of human bodies and therefore their vocal tract changes between birth and adult life between roughly $7 \mathrm{~cm}$ and $17 \mathrm{~cm}$. Furthermore, female speakers have on average shorter vocal tracts than male speakers, yielding higher formant frequencies. If interstellar life forms undergo a life cycle similar to life forms on Earth, during which they grow (which is very probable if we are dealing with biological life forms), and if these life forms have differences in body size due to what ever biological reasons, the physical characteristics of their communication signals will vary. Crucially, this will be true not only for acoustic signals generated by wind pipes, but also by other kinematic mechanisms, as well as by electromagnetic mechanisms, i.e. light emitters. 


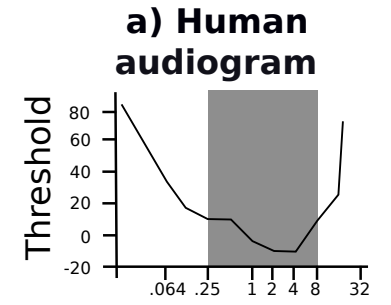

Frequency in $\mathrm{kHz}$ b) Absorption of electromagnetic frequencies

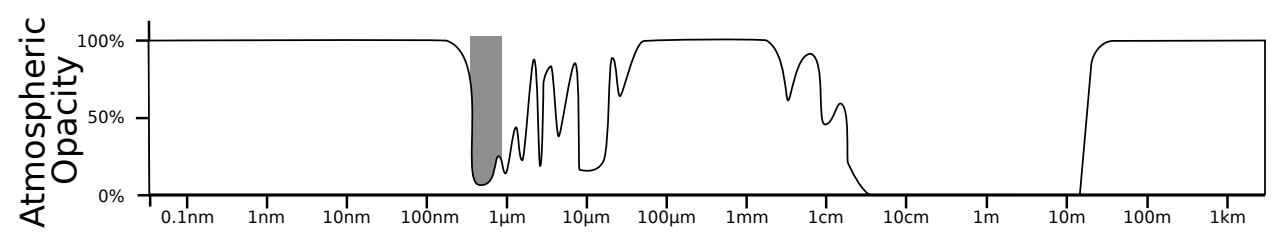

Wavelength

Fig. 4: a) Human audiogram. Frequencies on the $x$-axis. Perceptual threshold on $y$-axis in $d B$. The grey bar indicates perceptual optimum. b) Atmospheric opacity, indicating the absorption (y-axis) of electromagnetic frequencies (x-axis). The grey bar represents the human visual window. Figure according to Bothais (1987); Goody and Yung (1995).

Earth's atmospheric characteristics and human physiology allow humans to produce a signal ranging between $50 \mathrm{~Hz}$ and 20,000 $\mathrm{Hz}$ (Figure 4, a). Human pitch ranges from $70 \mathrm{~Hz}$ to $300 \mathrm{~Hz}$, vowels are encoded primarily within $250 \mathrm{~Hz}$ and 2,500 Hz, and consonants such as fricatives use frequencies up to $8,000 \mathrm{~Hz}$. Inspecting the human audiogram (Figure 4, a), the obvious conclusion arises that human hearing capabilities probably co-evolved with our speaking capabilities, which depend on the physical properties of our atmosphere. The optimal frequencies for a human are located between $250 \mathrm{~Hz}$ and $8,000 \mathrm{~Hz}$, exactly the frequency range in which human acoustic communication is located. More importantly, the increased sensitivity between $1000 \mathrm{~Hz}$ and 8000 $\mathrm{Hz}$ seems to compensate for the decrease in spectral amplitude in these frequency ranges (cf. Figure 3, a\&c). We therefore expect interstellar life forms to have adapted both production and perception to the physical characteristics of their home planet's atmosphere. This conclusion is not restricted to communication in the acoustic domain.

Like the acoustic signal, electromagnetic communication is highly constrained by the physical properties of a planet's atmosphere, i.e. its electromagnetic opacity (Figure 3, g). Earth's atmosphere, for example, provides only two windows for electromagnetic communication (Bothais, 1987; Goody and Yung, 1995). The first is located between a wavelength of $100 \mathrm{~nm}$ and $100 \mu \mathrm{m}$. It is for a small part of the first window that human and most animal vision evolved to be optimal (represented by the grey bar). The second window between a wavelength of roughly $1 \mathrm{~cm}$ and $10 \mathrm{~m}$ allows us to use radio waves for communication. In the case of Earth's atmosphere, the opacity is defined by the ratio of water vapor, oxygen, and ozone. Knowing the composition of an exoplanet's atmosphere will allow us to predict for which electromagnetic frequencies life forms on that planet have evolved. Likewise, this knowledge will allow us to predict whether we have common visual ranges. 


\section{Implications for the communication systems of extra-terrestrial life forms}

If interstellar life forms have evolved in different atmospheric conditions and have different sized bodies, but have similar learning mechanisms as the species of Earth, we can make certain predictions about the characteristics of their communication system. Firstly, the system will be highly adaptive under conditions of uncertainty. Secondly, the system will become significantly less adaptive with learning or as uncertainty decreases. Like Earth species, as learning approaches asymptote, it will become more difficult for these entities to adapt to new information, as long as event outcomes remain predictable.

Importantly, the system will downweight or unlearn cues that are not informative for predicting outcomes. This vital mechanism is what allows the life forms to ignore the noise in the system and learn to discriminate to a high level of sensitivity the cues that are important for communication of messages. This same mechanism also has the effect that cues in the signal that are detectable with the life form's sensory system will end up not being discriminated. The life form has learned to ignore the variation in the cues, as in the simulation of perception of interstellar vowels by human listeners. Note that events and objects in the world provide outcomes that enable discrimination learning of the speech signal (a 'dog' is not a 'dock'). Linguistic labels in turn provide outcomes for discrimination learning of events and objects in the world (a dog is not a cat). Communication is predictive about the world, and the world is also predictive about communication events. Each aids and influences the learning of the other.

Using the values obtained above for species that produce speech in the same way as humans, but have long, $30 \mathrm{~cm}$ vocal tracts, we simulate an interstellar species learning two vowel categories. Although we expect an unimaginably large degree of variation is possible in the types of communcation signals across interstellar species, for the sake of illustration, we consider only tiny deviations from the characteristics of Earth's languages.

Note that this analysis assumes that the interstellar species experience time in a linear fashion as on Earth. Events earlier in time can be used to predict upcoming events, but not vice versa. If this is not the case, we might expect quite different learning mechanisms, such as for example, Hebbian learning, in which learning occurs through simple co-occurrence of stimuli, rather than prediction of outcomes from cues. 


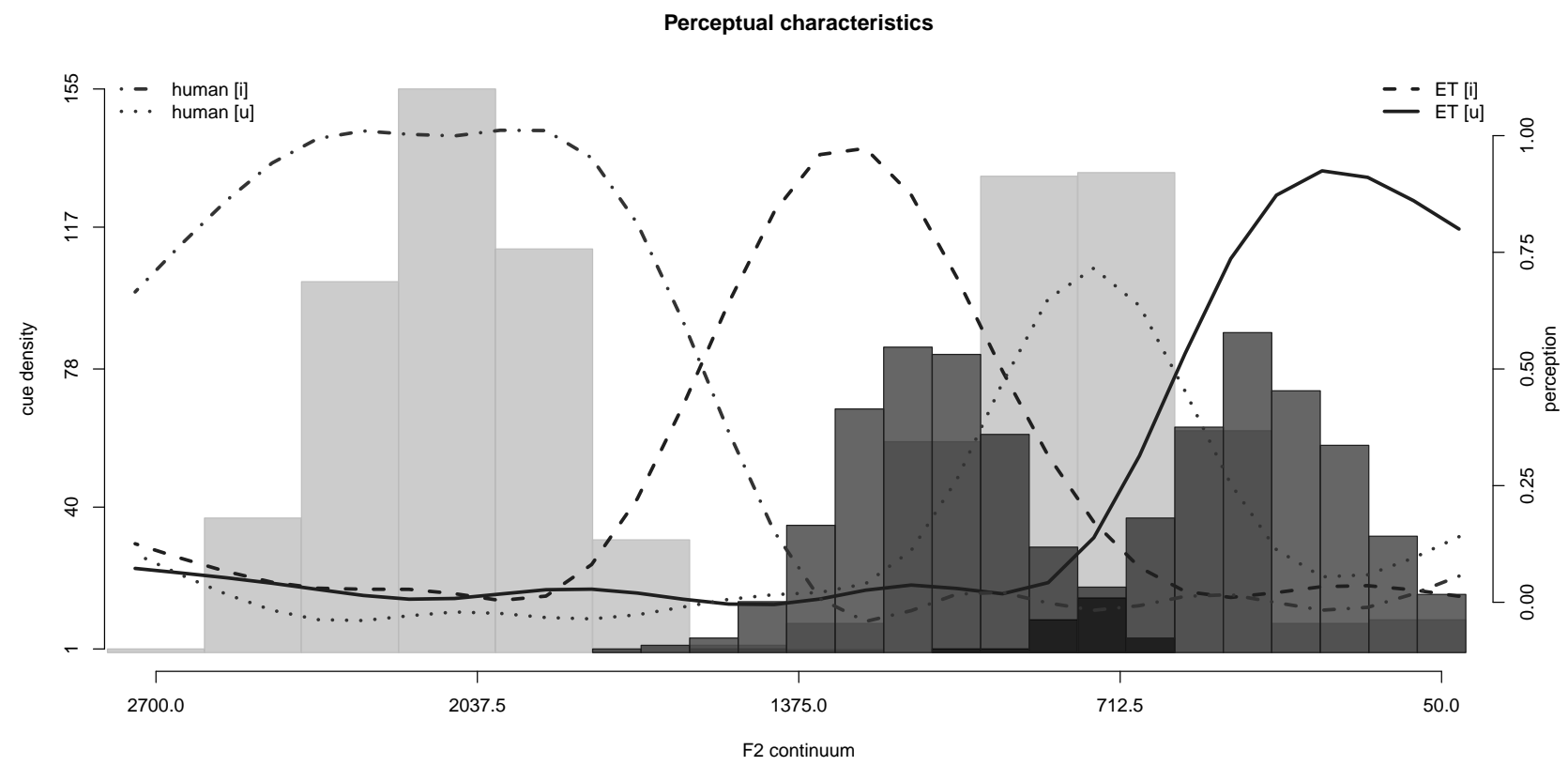

Fig. 5: Figure illustrates how perceptual characteristics (lines, left y-axis) develop form different distributions of cues (bars, right $y$-axis) along a given continuum (x-axis). Note that the $x$-axis is inverted to mirror the vowel space in Figure 2. Light grey bars represent the distribution of F2 cues for humans, with higher frequencies being interpreted as [i] and lower frequencies as [u]. Dark grey bars represent distribution of F2 for extraterrestrial life forms.

Error-driven learning is also most expected when there is a sufficient degree of uncertainty in the organism's world to create the necessity of unlearning non-discriminative cues or noise in the signal. Note also that this simulation addresses the specific situation where the frequency range partially overlaps the human speech frequency range. Different results would be expected if there were no overlap or if the communication signal consisted of entirely different cues (such as electromagnetic, for example, or acoustic cues not familiar to the listener).

Using the Rescorla-Wagner learning equations (Rescorla \& Wagner, 1972), a computational formalization of error-driven, discriminative learning, we simulated the perceptual characteristics of the $[i]$ and $[\mathrm{u}]$ vowels ${ }^{4}$, i.e. to what extent the various cues are perceived as one or the other vowel. The equations learn to discriminate the vowels by formant cue due to the covariance structure in the data. This simulation is based on vowel categories that are produced with the same articulatory gestures as human (e.g. English) [i] and [u]. The first simulation models human learning and is trained on human vowels. The F2 cue for these vowels clusters in two normal distributions with peaks located at $800 \mathrm{~Hz}$ and $2100 \mathrm{~Hz}$ (Figure 5, light grey bars). The second simulation is trained 
on the values of extra-terrestrial vowels [i] and [u] which cluster around $400 \mathrm{~Hz}$ and $1500 \mathrm{~Hz}$ (Figure 5, dark grey bars). In this tiny, toy example, the cues are the spectral frequencies and there are only two possible outcomes, $[i]$ and $[\mathrm{u}]$ for each species. ${ }^{5}$ The lines represent the activation level for each vowel for the humans and for the interstellar intelligence. [i]For each different vowel outcome, activation is high at different points along the F2 continuum depending on the training.

The dashed-dotted (for [i]) and dotted lines (for [u]) show that the human perception depends on the human cue distribution. Activation decreases towards the edges of the continuum, resulting in two perceptual categories with a shift from one vowel to the other located roughly at $1350 \mathrm{~Hz}$. A similar pattern can be observed for the simulated interstellar life forms. However, the activation peaks are located in lower frequency range, due to the cues in their training. The critical result is for the human $[\mathrm{u}]$ (dotted line). At the points along the F2 continuum that activate two different vowels [i] and $[\mathrm{u}]$ for the interstellar life form, there is only activation of $[\mathrm{u}]$ for the human listeners. Therefore, both alien vowel categories are likely to be perceived as $[\mathrm{u}]$ - even if perhaps not good instances of $[\mathrm{u}]$. Both of the above cases illustrate perception after training in the native communication systems. If there were sufficient information available to provide error feedback that this initial perception or prediction was sometimes incorrect, then learning through prediction error could occur. The listeners might eventually learn to discriminate the new vowels.

\section{Conclusion}

Error-driven learning is a pervasive mechanism that functions across the spectrum of Earth's species for adaptively learning about the world. Although late to the table in application to human learning, it is proving to be effective an explanatory tool there too. Error-driven learning has solved much of the mystery regarding how humans are able to learn such a complex system as human language. We hypothesise that it may also play a role in other interstellar species learning about their world, including their communication systems. If so, this would have certain implications for their perception and communication, particularly in relation to effects of uncertainty and familiar vs. unfamiliar cues. We have also demonstrated that, even when articulation is identical to human articulation, quite significant effects on an acoustic signal emerge from only minor changes in body size or atmospheric conditions. We have discussed some practical implications for researchers preparing materials for interstellar communication. Language materials on their own probably have very little information value. From our perspective, language is not defined by universal sequences of abstract categories but emerges through ongoing learning from the covariance structure, where different kinds of cues - acoustic, visual, tactile, olfactory or other sensory information - converge

5 This is a gross oversimplification. We do not assume that vowel categories are learned with vowel labels in the real world, but rather that speech co-occurs with events and the presence of various objects and so on, as discussed above. However, we restrict ourselves to these items for the purposes of illustration. 
on different world-related events. That is, concepts arise through the experience of language in context. Experiencing the signals for these concepts with additional information such as audio recordings and images of them - or in whatever sensory or other modality is appropriate - will allow extra-terrestrial learners (or their equivalent of machine learning methods) to learn to discriminate which acoustic cues in the signal discriminate which images. If the METI project wants to create a common code, rather than broadcasting random, unrelated audio and video samples (see Harbour, this volume), it must compile a selection of distributionally structured and contextually dependent information provided in multi-modal channels.

\section{References}

Altmann, G. 1980. “Prolegomena to Menzerath’s Law.” Glottometrika 2:1-10.

Arnold, D., F. Tomaschek, K. Sering, M. Ramscar, and R. H. Baayen. 2017. "Words from spontaneous conversational speech can be recognized with human-like accuracy by an error-driven learning algorithm that discriminates between meanings straight from smart acoustic features, bypassing the phoneme as recognition unit." PLOS ONE.

Baayen R. H., Chuang Y., Shafaei-Bajestan E. and Blevins J. P. 2019. “The discriminative lexicon: A unified computational model for the lexicon and lexical processing in comprehension and production grounded not in (de) composition but in linear discriminative learning.” Complexity.

Baayen, R. H., P. Milin, D. F. Đurđević, P. Hendrix, and M. Marelli. 2011. “An amorphous model for morphological processing in visual comprehension based on naive discriminative learning." Psychological review 118 (3): 438-481.

Baayen R. H., Shaoul C., Willits J., Ramscar M. (2016), Comprehension without segmentation: a proof of concept with naive discriminative learning. Language, Cognition and Neuroscience (31) 1, p106ff.

Bothais, L. 1987. Radio Wave Propagation. McGraw-Hill Book Company, New York.

Chung, Kevin K. H. 2003. "Effects of Pinyin and first language words in learning of Chinese characters as a second language." Journal of Behavioral Education 12 (3): 207-223.

Clayards, M., M. K. Tanenhaus, R. N. Aslin, and R. A. Jacobs. 2008. "Perception of speech reflects optimal use of probabilistic speech cues.” Cognition 108 (3): 804-809.

Doherty, John A. 1985. “Temperature Coupling and 'Trade-Off' Phenomena in the Acoustic communicative system of the Cricket, Gryllus Bimaculatus De Geer (Gryllidae).” Journal of 
Experimental Biology 114 (1): 17-35.

Fant, G. 1960. Acoustic theory of speech production. De Gruyter.

Fitch, W. Tecumseh. 1997. "Vocal tract length and formant frequency dispersion correlate with body size in rhesus macaques." The Journal of the Acoustical Society of America 102 (2): 1213-1222. eprint: https://doi.org/10.1121/1.421048. https://doi.org/10.1121/1.421048.

Flege, J. E., \& MacKay, I. R. 2004. "Perceiving vowels in a second language.” Studies in second language acquisition, 26(1), 1-34.

Fosler-Lussier, Eric, and Nelson Morgan. 1999. "Effects of speaking rate and word frequency on pronunciations in convertional speech.” Speech Communication 29 (2-4): 137-158.

http://www.sciencedirect.com/science/article/pii/S0167639399000357.

Fuchs, S., R. Winkler, and P. Perrier. 2008. “Do speakers' vocal tract geometries shape their articulatory vowel space?," Proceedings of the 8th International Seminar on Speech Production, ISSP'08, Strasbourg : France. 333-336.

Gay, Thomas. 1978. "Effect of speaking rate on vowel formant movements." The Journal of the Acoustical Society of America 63 (1): 223-230.

Gervain, Judit. 2015. "Plasticity in early language acquisition: the effects of prenatal and early childhood experience." Current opinion in neurobiology 35:13-20.

Gluck, M. A., \& Bower, G. H. (1988). From conditioning to category learning: an adaptive network model. Journal of Experimental Psychology: General, 117(3), 227.

Goody, R. M., and L. Yung. 1995. Atmospheric Radiation. Oxford University Press.

Gray, D. A. 1997. "Female house crickets,Acheta domesticus, prefer the chirps of large males." Animal Behaviour 54 (6): 1553-1562.

Hirata, Yukari. 2004. "Effects of speaking rate on the vowel length distinction in Japanese." Journal of Phonetics 32 (4): 565-589.

Hull, C. L. (1943). Principles of behavior. New York: Appleton-Century-Crofts.

Kalman, R. E. A new approach to linear filtering and prediction problems. J. Basic Eng. Trans. ASME 82, 35-45 (1960).

Kamin, Leon J. 1968. “Attention-like processes in classical conditioning.” In Miami symposium on the prediction of behavior, edited by M. R. Jones, 9-31. Miami: Miami University Press.

Knox A., Markx J., How E., Azis A., Hobaiter C., van Veen,F. J., Morrogh-Bernard H. 2019. “Gesture Use in Communication between Mothers and Offspring in Wild Orang-Utans (Pongo 
pygmaeus wurmbii) from the Sabangau Peat-Swamp Forest, Borneo.” International Journal of Primatology, 1-24.

Landauer, T. K., \& Dumais, S. T. (1997). A solution to Plato's problem: The latent semantic analysis theory of acquisition, induction, and representation of knowledge. Psychological review, 104(2), 211.

Leemann, Adrian, Volker Dellwo, Marie-José Kolly, and Stephan Schmid. 2012. "Rhythmic variability in Swiss German dialects.” In Speech Prosody 2012.

MacLean, DJ. 1966. "Analysis of Speech in a Helium-Oxygen Mixture under Pressure.” The Journal of the Acoustical Society of America 40 (3): 625-627.

McMurray, B., R. N. Aslin, and J. C. Toscano. 2009. "Statistical learning of phonetic categories: insights from a computational approach.” Developmental Science 12 (3): 369-378.

Magen, H. S. 1997. "The extent of vowel-to-vowel coarticulation in English." Journal of Phonetics, no. 25 : 187-205.

Maye, J., Werker, J. F., Gerken, L., 2002. Infant sensitivity to distributional information can affect phonetic discrimination. Cognition 82 (3).

Meyer, J. 2004. Bioacoustics of human whistled languages: an alternative approach to the cognitive processes of language. Anais da Academia Brasileira de Ciências, 76(2), 406-412.

NASA. n.d. https://www.grc.nasa.gov/WWW/K-12/rocket/machu.html.

Nixon, J. S. 2018. "Effective acoustic cue learning is not just statistical, it is discriminative." In Interspeech 2018 - 19th Annual Conference of the International Speech Communication Association, 1447-1451. Hyderabad, India, September.

--- . 2020. "Of mice and men: speech acquisition as discriminative error-driven learning, not just statistical tracking.” Accepted for publication in Cognition 197, 104081.

Nixon, J. S., J. van Rij, P. Mok, R. H. Baayen, and Y. Chen. 2016. "The temporal dynamics of perceptual uncertainty: eye movement evidence from Cantonese segment and tone perception." Journal of Memory and Language 90:103-125.

Nixon, J. S., and Best, C. T. 2018. "Acoustic cue variability affects eye movement behaviour during non-native speech perception." In Proceedings of the 9 International Conference on Speech Prosody, 493-497. Poznan, Poland, June.

Nixon, J. S., Boll-Avetisyan, N. Lentz, T. O., van Ommen, S. Keij, B. Çöltekin, Ç., Liu, L. and van Rij, J. 2018. "Short-term exposure enhances perception of both between- and within-category acoustic information." In Proceedings of the 9th International Conference on Speech Prosody. 
Nyst V.A.S. (2013), The Significance of African Sign Languages for African Linguistics and Sign Language Studies. In: Orie O. O., Sanders K.W. (Eds.) Selected Proceedings of the 43rd Annual Conference on African Linguistics: Linguistic Interfaces in African Languages. Somerville, MA, USA: Cascadilla Proceedings Project. 77-81.

Nyst, V. (2015). The Sign Language Situation in Mali. Sign Language Studies, Volume 15, Number 2, pp. 126-150.

Öhman, S.E.G. 1966. “Coarticulation in VCV Utterances: Spectrographic Measurements.” Journal of the Acoustical Society of America 39 (151): 151-168.

Popov, A. V., and V. F. Shuvalov. 1977. "Phonotactic behavior of crickets." Journal of comparative physiology 119, no. 1 (January): 111-126.

Ramscar, M., M. Dye, J.W. Gustafson, and J. Klein. 2013. "Dual routes to cognitive flexibility: Learning and response conflict resolution in the dimensional change card sort task." Child Development 84:1308-1323.

Ramscar, M., P. Hendrix, C. Shaoul, P. Milin, and R. H. Baayen. 2014. "The Myth of Cognitive Decline: Non-Linear Dynamics of Lifelong Learning." Topics in Cognitive Science 6 (1): 5-42. Ramscar, M., C. C. Sun, P. Hendrix, and R. H. Baayen. 2017. “The Mismeasurement of Mind: LifeSpan Changes in Paired-Associate-Learning Scores Reflect the "Cost" of Learning, Not Cognitive Decline.” Psychological Science.

Ramscar, M., and D. Yarlett. 2007. "Linguistic Self-Correction in the Absence of Feedback: A New Approach to the Logical Problem of Language Acquisition.” Cognitive Science 31 (6): 927-960.

Ramscar, M., D. Yarlett, M. Dye, K. Denny, and K. Thorpe. 2010. “The Effects of Feature-LabelOrder and their implications for symbolic learning." Cognitive Science 34 (6): 909-957.

Ramscar M. 2019. “Source codes in human communication.” https://psyarxiv.com/e3hps.

Rattray, R. S. (1923). The drum language of West Africa. African Affairs, 22(LXXXVII), 226-236.

Rescorla, R., and A. Wagner. 1972. "A theory of pavlovian conditioning: Variations in the effectiveness of reinforcement and nonreinforcement." In Classical conditioning II: Current research and theory, edited by A. H. Black and W.F. Prokasy, 64-69. Appleton Century Crofts, New York.

Shannon, C. E. (1948). “A mathematical theory of communication.” Bell system technical journal, 27(3), 379-423.

Slobodchikoff, C. N., and J. Placer. 2006. “Acoustic structures in the alarm calls of Gunnison's 
prairie dogs.” The Journal of the Acoustical Society of America 119 (5): 3153-3160.

Soto, F. A., \& Wasserman, E. A. (2010). Error-driven learning in visual categorization and object recognition: A common-elements model. Psychological review, 117(2), 349.

Spence K. W . (1956). Behavior theory and conditioning. New Haven, CT: Yale University Press. Tomaschek, F., D. Arnold, Franziska Bröker, and R. H. R. Baayen. 2018. “Lexical frequency codetermines the speed-curvature relation in articulation." Journal of Phonetics.

Tomaschek, F., and A. Leeman. 2018. "The size of the tongue movement area affects the temporal coordination of consonants and vowels - A proof of concept on investigating speech rhythm." JASA-EL.

Tomaschek, F., Plag, I., Ernestus, M., \& Baayen, R. H. 2019. "Phonetic effects of morphology and context: Modeling the duration of word-final S in English with naïve discriminative learning." Journal of Linguistics, 1-39.

Tomaschek, F, H. Truckenbrodt, and I. Hertrich. 2011. “German Vowel Quantity: Categorical Perception or Perceptual Magnet Effect?” In Proceedings of the 17th International Congress of Phonetic Sciences (ICPhS XVII, Hong Kong), 2002-2005.

Tomaschek, F., H. Truckenbrodt, and I. Hertrich. 2015. "Discrimination sensitivities and identification patterns of vowel quality and duration in German /u/ and /o/ instances." In Trends in phonetics and phonology. Studies from German speaking Europe, edited by A. Leemann, M.-J. Kolly, S Schmid, and V. Dellwo. Frankfurt

Tomaschek, F., B. V. Tucker, R. H. Baayen, and M. Fasiolo. 2018. "Practice makes perfect: The consequences of lexical proficiency for articulation." Linguistic Vanguard.

Tomaschek, F., B. V. Tucker, M. Wieling, and R. H. Baayen. 2014. "Vowel articulation affected by word frequency." In Proceedings of the 10th ISSP, 425-428. Cologne.

Tomaschek, F., M. Wieling, D. Arnold, and R. H. Baayen. 2013. "Word frequency, vowel length and vowel quality in speech production: An EMA study of the importance of experience." In Proceedings of the Interspeech. Lyon.

Waelti P., Dickinson A., Schultz W. 2001. "Dopamine responses comply with basic assumptions of formal learning theory.” Nature, 412, 43-48.

Weirich, M., and S. Fuchs. 2006. "Palatal morphology can influence speaker-specific realizations of phonemic contrasts." Journal of Speech, Language, and Hearing Research 56:1894-1908.

Werker, J. F., and R. C. Tees. 1984. “Cross-language speech perception: Evidence for perceptual 
reorganization during the first year of life." Infant behavior and development 7 (1): 49-63.

Widrow, G. \& Hoff, M. E. Adaptive switching circuits. IRE Western Electron. Show Convention, Convention Record Part 4, 96-104 (1960).

Winkler, R., S. Fuchs, and P. Perrier. 2006. "The relation between differences in vocal tract geometry and articulatory control strategies in the production of French vowels: Evidence from MRI and modeling," In 8th International Seminar on Speech Production, ISSP'08, Strasbourg : France. 509-516.

Wittgenstein, L. (1953). Philosophical investigations [Philosophische Untersuchungen]. Yashiro, Kohtaro, and Kenji Takada. 2005. "Model-based analysis of jaw-movement kinematics using jerk-optimal criterion: simulation of human chewing cycles."

Yoshida, K. A., F. Pons, J. Maye, and J. F. Werker. 2010. “Distributional phonetic learning at 10 months of age.” Infancy 15 (4). 